\title{
Image Registration using Combination of GPOF and Gradient Method for Image Super Resolution
}

\author{
Niyanta Panchal \\ Computer Science \& Engg. Dept, \\ Parul Institute Of Technology, \\ Waghodia,Vadodara
}

\author{
Ankit Prajapati \\ Computer Science \& Engg, Dept \\ Faculty of Engineering \& \\ Technology, At. Isroli, Po.Afwa
}

\author{
Bhailal Limbasiya \\ Computer Science \& Engg. Dept, \\ Parul Institute Of Technology, \\ Waghodia,Vadodara
}

\begin{abstract}
Super Resolution implementation using multi-frame super resolution has been an expensive topic in the literature. Multiframe Super-Resolution is to generate the high-resolution image from multiple low-resolution images perspectives of a same scene. Most important part of multi-frame Superresolution is Image Registration; that estimates the translation, rotation and scaling parameters and also aligns images. In this paper, they propose a combination of Gaussian Pyramid Optical Flow (GPOF) Registration method and Gradient method for constructing Super Resolution image. In the proposed approach, they focus on the movement model of the image registration GPOF, which reach the sub-pixel and allows for the large pixel motions, while keeping the size of image neighborhood relatively small. And apply Gradient method, which can accurately perform precise registration with the amount of image movement is small between the two images; it can get the one reconstructed image. They get the better results compares with the others registration methods. And lastly, they apply Discrete Wavelet Transform (DWT) image interpolation algorithm; they can get the high resolution image. Experiment results show that the HR image by their proposed method have much higher quality than other methods.
\end{abstract}

\section{Keywords}

Super-Resolution; Gaussian Pyramid Optical Flow; Gradient method; Discrete Wavelet Transform;

\section{INTRODUCTION}

Image Super Resolution has been an expensive and most wide topic of research area. In many of the application likes Medical imaging, Satellite imaging, Remote imaging, Video surveillance, enlarging consumer photograph for higher quality imaging with high resolution images are required and most important desired. In that various image sensors are useful for this application. But, high-resolution sensor is very expensive. So, they need increase the current resolution by two ways, either reducing the pixel size or by increasing the chip size. However it can produce low resolution images because of it has some limitations. Therefore, a new method is required to increase the resolution of the image.

Super-Resolution (SR) is to obtain a high-resolution (HR) image using multiple observed low-resolution (LR) images by down-sampling, de-blurring, and de-noising. Where, the LowResolution (LR) image represents low pixel quality and it provides less accurate details. High-Resolution (HR) image represents high pixel quality and it provides more accurate details.

In the Super-resolution techniques, that have classifies into two major parts: Frequency Domain approach, and Spatial
Domain approach. Frequency Domain approach, which can perform Fourier transform of an image. These methods are simple and computationally cheap, they are extremely sensitive to model error, limiting their use and Spatial Domain approach, which can perform directly on pixel and it is also more popular method. These methods are computationally expensive.

Now, for the technical implementation of Super-Resolution in two ways: Single-frame and Multi-frame image SuperResolution. Single-frame Super-Resolution methods to generate a Single High-Resolution image from single degraded or noisy or blurred image. Multi-frame SuperResolution is to produce the high-resolution (HR) image from multiple low-resolution (LR) images with same scene.

Most important part of multi-frame super resolution is image registration. In recently researches topic, some of the image registration methods for image super resolution like optical flow method [6], PCA based method [4], and Gradient methods [4]. In such that image registration using two images, which is achieves small amount of motion for shift or rotation parameter. But they cannot perform the scale parameter. Optical flow method cannot perform the scale parameter between the two images. PCA based method The PCA based registration can perform the large amount of motion for shifting, rotation and scaling between the two images. In the Gaussian Pyramid Optical Flow (GPOF) based method, which allows to perform the large pixel motions for the translation and scale between the two images, while keeping the size of image neighborhood relatively small and Gradient Method, which can measure image movement relatively small pixel motion. Therefore, our paper proposes combination of GPOF method and Gradient method for the image super resolution. Here, first we apply the GPOF method for large amount of movement parameter between the two images and then apply Gradient method for small amount of movement parameter. And lastly we apply the image interpolation using DWT; we achieve the high resolution image. And we compare image registration methods for image super resolution; we achieve the better results.

This paper is organized as follows. In Section 2, we describe Multi-frame Image Super-Resolution. Section 3 represents Proposed Work. Section 4 represents Experiments Results. Section 5 represents Conclusion.

\section{MULTI-FRAME IMAGE SUPER RESOLUTION}

Multi-frame Super-Resolution is to generate the highresolution (HR) image from multiple low-resolution images perspectives of a same scene and also increase spatial resolution by fusing information. 
There are main two steps for multi-frame image SuperResolution, 1) Image Registration, which is performed first in order to align the LR images as accurately as possible, and also estimate movement parameters such as shift, rotation and scale, 2) Image Interpolation, which is done to get highresolved image. So, Image registration plays accurate role in image reconstruction process.

Image Registration is the most important method for multiframe image super resolution. It is used for motion estimation likes shift, rotation and scale estimation. The main goal for image registration is also aligning the images to decrease the difference between the reference image and the changing image.

There are three types of registration parameters: Translation (shift) parameter, which calculates horizontal and vertical displacement between the reference image and the changing image; Rotation parameter, which calculates shift angle between two images; Scale parameter, which calculates size variance in the same object. No. of image registration are given in below.

\subsection{GPOF Registration Method ${ }^{[3]}$}

For super-resolution reconstruction with image registration methods Gaussian Pyramid Optical Flow (GPOF), which can perform the sub-pixel precision and enable to allows large pixel motions for the translation and scaling parameter. it means pixel points move very far, while keeping image neighborhood (integration window) of size relatively small.

In that basic idea of GPOF method first build a Gaussian pyramid, and then compute the optical flow displacement between two images.

In Gaussian pyramid, first we define the no. of levels $\mathrm{K}$ likes maximal number of levels 6 . Next, for build a pyramid g1 as base image, we down sample given base level image using anti aliasing low-pass filter at each level till up to the highest level K. we can get the low resolution image at each level.

Then next step, between the two low resolution frames, it computes displacement $d$ at each level of the pyramid. First initial value of $\mathrm{d}=0$ for first two frame images g1 and $\mathrm{g} 2$ at all level K. It starts computing from the upper level till down to the lowest level of the pyramid.

The advantage of Gaussian Pyramid Optical Flow (GPOF) registration is that it can reduce the computing complex process and the algorithm implementation speed is fast because each residual optical flow vector can be kept very small while computing a large overall pixel displacement vector [3].

\subsection{Gradient Registration Method ${ }^{[4]}$}

Gradient based registration method can accurately measure translation and rotation parameter with the two images original image and image for measuring movement parameters.

In this method, first is to optimize the cost function, which measures similarity between the original image and the registered images by the mutual information measurement, and second then it can be implemented by the steepest descent method. In this procedure, they initialize the value of movement parameter. If real movement parameters are near to the initial value of parameters, so they can get the fast optimized parameters. If real movement parameters are far to the initial value of parameters, so we can use optimized solver with gradient method may be discover the minimum point and cannot get the real parameter [4].

\section{PROPOSED WORK}

In this paper, image registration using combination of GPOF and Gradient method gives more accurate movement parameters. First, we apply the GPOF registration method. It is to perform first build a Gaussian pyramid for each frame of the LR observed Sequence. Then compute the optical flow d which is regarded as the displacement from image g2 to image g1 in each level of the pyramid between two observed images. It starts computing from the upper level till down to the lowest level of the pyramid. GPOF, which reach the sub-pixel and can measure accurate parameters for the large pixel motion.

Second, we apply Gradient based registration method. It can accurately measure translation and rotation parameter with the original image and registered image. Between these two images content are similar, but the amount of the movement parameter is small. It also can perform precise registration with minor image movement. And we can get the one output image.

Lastly, we apply the image interpolation algorithms using DWT [5]. There are number of steps for interpolation. They interpolate an original image (I) of resolution $\mathrm{m} \times \mathrm{n}$ to an image (I' DWT) of dimension resolution $2 \mathrm{~m} \times 2 \mathrm{n}$ all pixel elements as zero. In the first step, the image is divided into four sub bands: LL, HL, LH and HH by DWT. The HL and LH sub bands contain edge information in horizontal and vertical directions, respectively.

Then, second step is to form a new wavelet coefficient image a matrix I 'DWT of size $2 m \times 2 n$ with the pixel size zero. That image is said as virtual DWT image, whose LL sub band is nothing but the original input image I with each pixel multiplied by a scaling factor $\boldsymbol{s}$. The matrix is said to a ILL. This scale factor $s$ is set equal to the square of the DC gain of the selected analysis low-pass filter. Depending upon the results of implementation of the discrete wavelet transform (DWT) first may choose a different DC gain for the analysis low-pass filter and Nyquist gain for the analysis high-pass filter, or we choose other filters.

Then next step, by using the low pass filter in each column followed to apply the high-pass wavelet filter in each row of I. we get the resultant matrix IHL. By this IHL, replace the topright quadrant of the matrix I'DWT.

Then next step, by using the high pass filter in each column followed to apply the low-pass wavelet filter in each row of I. we get the resultant matrix ILH. By this ILH, replace the bottom-left quadrant of the matrix I'DWT. Apply the inverse DWT on matrix I'DWT to produce resultant matrix I'. And we get the reconstructed image or high resolution image.

\section{EXPERIMENT RESULTS}

For the experiment results, we have to use the different quality measure parameters such as MSE, PSNR and SSIM to evaluate proposed work.

Mean-Squared Error (MSE) is simply measure the squared error between the original image and the reconstructed image. The MSE can be expressed as [13],

$$
M S E=\frac{1}{m n} \sum_{i=0}^{m-1} \sum_{j=0}^{n-1}[I(i, j)-K(i, j)]^{2}
$$


Where, I and $\mathrm{K}$ represent the mxn matrices of image and comparison of images. For original image I, I (i, j) represents the value of pixel $(i, j)$ and for reconstructed image $K, K(i, j)$ represents the value of pixel $(i, j)$.

The Peak Signal-to-Noise Ratio (PSNR) is defined as a measuring of quality of reconstructed image and also comparing with original image.The PSNR can be expressed as [13]:

$$
\text { PSNR }=20 . \log _{10}\left(\frac{\mathrm{MAX}_{\mathrm{I}}^{2}}{\sqrt{\mathrm{MSE}}}\right)
$$

Here, MAX $_{I}$ performs highest value of pixel of the image. Mostly the pixels are represented 8 bits per sample; this is 255. And MSE performs error between the two images. The PSNR expressed in decibels.

SSIM measures contrast, structure of an image, compares variance and covariance between the original image and reconstructed image. The SSIM can be expressed as [13]:

$$
\operatorname{SSIM}(X, Y)=\frac{\left(2 \mu_{\mathrm{x}} \mu_{\mathrm{y}}+\mathrm{c}_{1}\right)\left(2 \sigma_{\mathrm{xy}}+\mathrm{c}_{2}\right)}{\left(\mu_{\mathrm{x}}^{2}+\mu_{\mathrm{y}}^{2}+\mathrm{c}_{1}\right)\left(\sigma_{\mathrm{X}}^{2}+\sigma_{\mathrm{y}}^{2}+\mathrm{c}_{2}\right)}
$$

Where, $\mathrm{x}$ and $\mathrm{y}$ are sub images of $\mathrm{X}$ and $\mathrm{Y}$, and $\mu \mathrm{x}, \mu \mathrm{y}$ are the average of $\mathrm{x}, \mathrm{y} . \sigma \mathrm{x}, \sigma \mathrm{y}$ are standard deviations of $\mathrm{x}, \mathrm{y} . \mathrm{C} 1$ is set to $\mathrm{C} 1=(0.01 * 255)^{\wedge} 2$ and $\mathrm{C} 2$ is set to $\mathrm{C} 2=(0.01 * 255)^{\wedge} 2$.
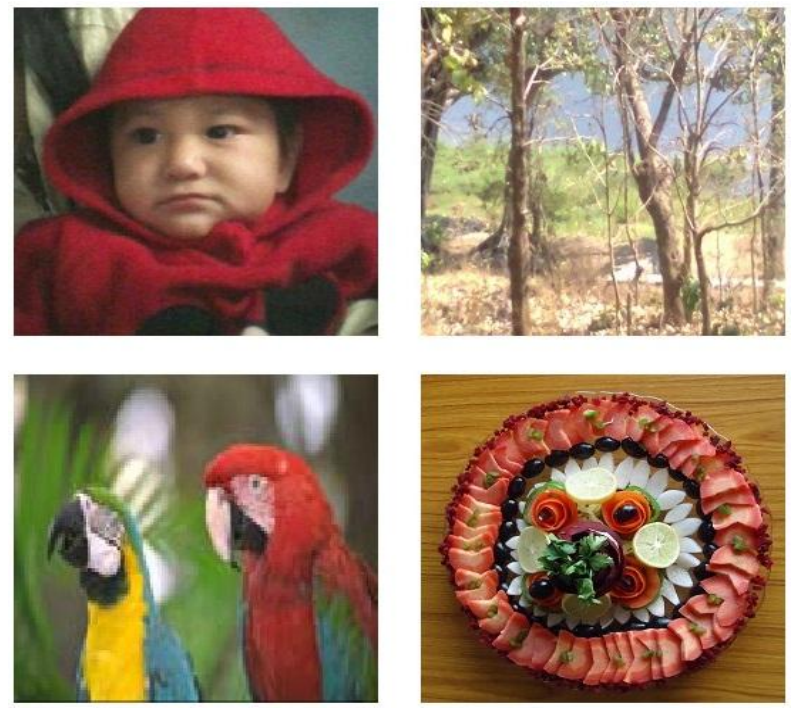

Figure 1: Input images with boy, nature, parrot and salad disc

We have considered for our experiment results in this 4 input images likes boy, nature, parrot, and salad disc in Figure 1. The size of all 4 input images is $256 \times 256$.

For the proposed algorithm, GPOF based registration method using the 4 input images are shown in Figure 2.
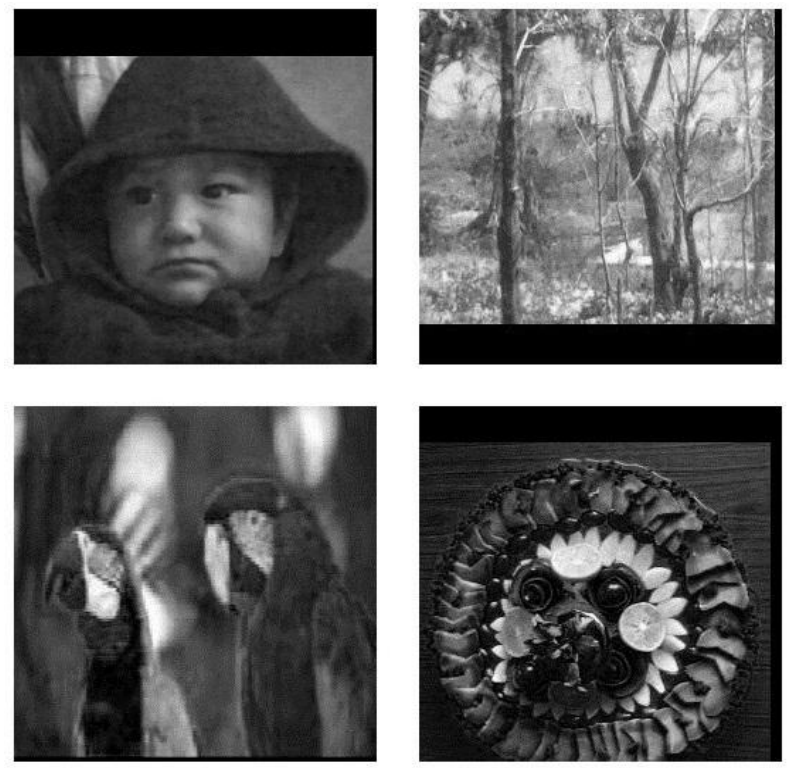

Figure 2: GPOF based registration method with translation image

For apply gradient based registration method, output of the GPOF image and the reference image using steepest decedent method. They get the optimized reconstructed image shown in Figure 3.
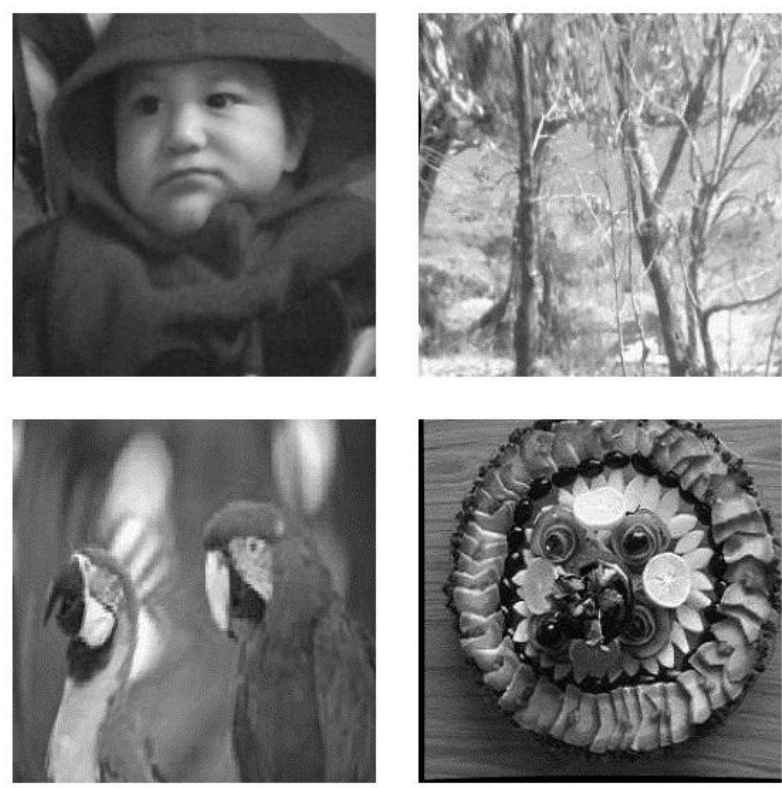

Figure 3: Gradient Based image registration with reconstructed image.

And lastly, Image interpolation using discrete wavelet transform, we get the High resolution image or reconstructed image for all 4 input images shown in Figure 4: shows the reconstructed image or high resolution image.

Implementation of GPOF registration method is also measures the translation parameters. Compute the displacement of the two images and estimation the movement parameter. And results give in Table 1. 
Image Quality measurement of PSNR, MSE and SSIM is defined between the two images. Experiment results of my proposed algorithm shown in Table 2.

Table 1. Displacement of the GPOF image

\begin{tabular}{|c|c|c|}
\hline $\begin{array}{l}\text { Movement } \\
\text { Parameter }\end{array}$ & \multicolumn{2}{|c|}{ Displacement } \\
\hline Translation & U & V \\
\hline
\end{tabular}
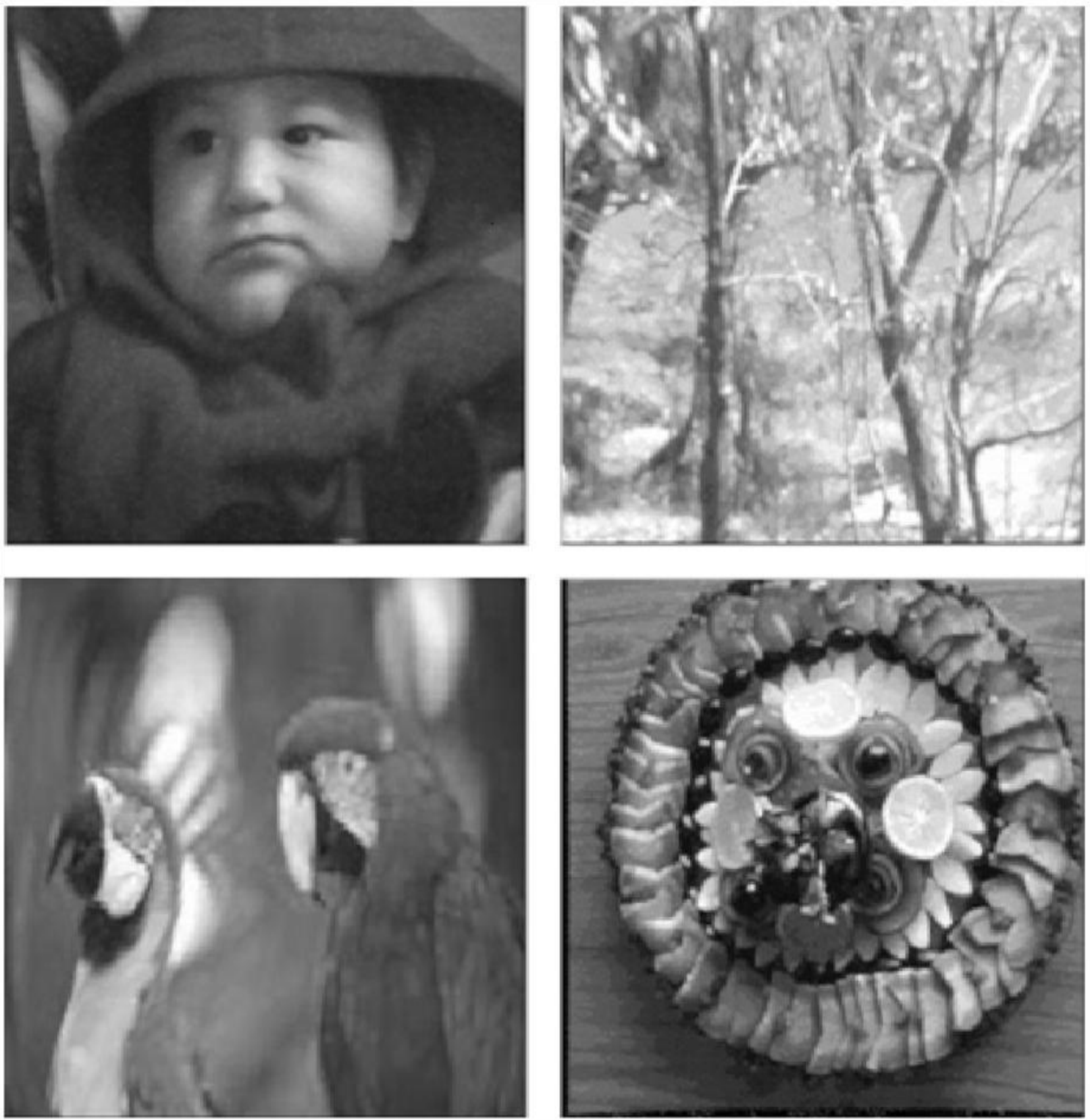

Figure 4: Reconstructed images or High-Resolution images 


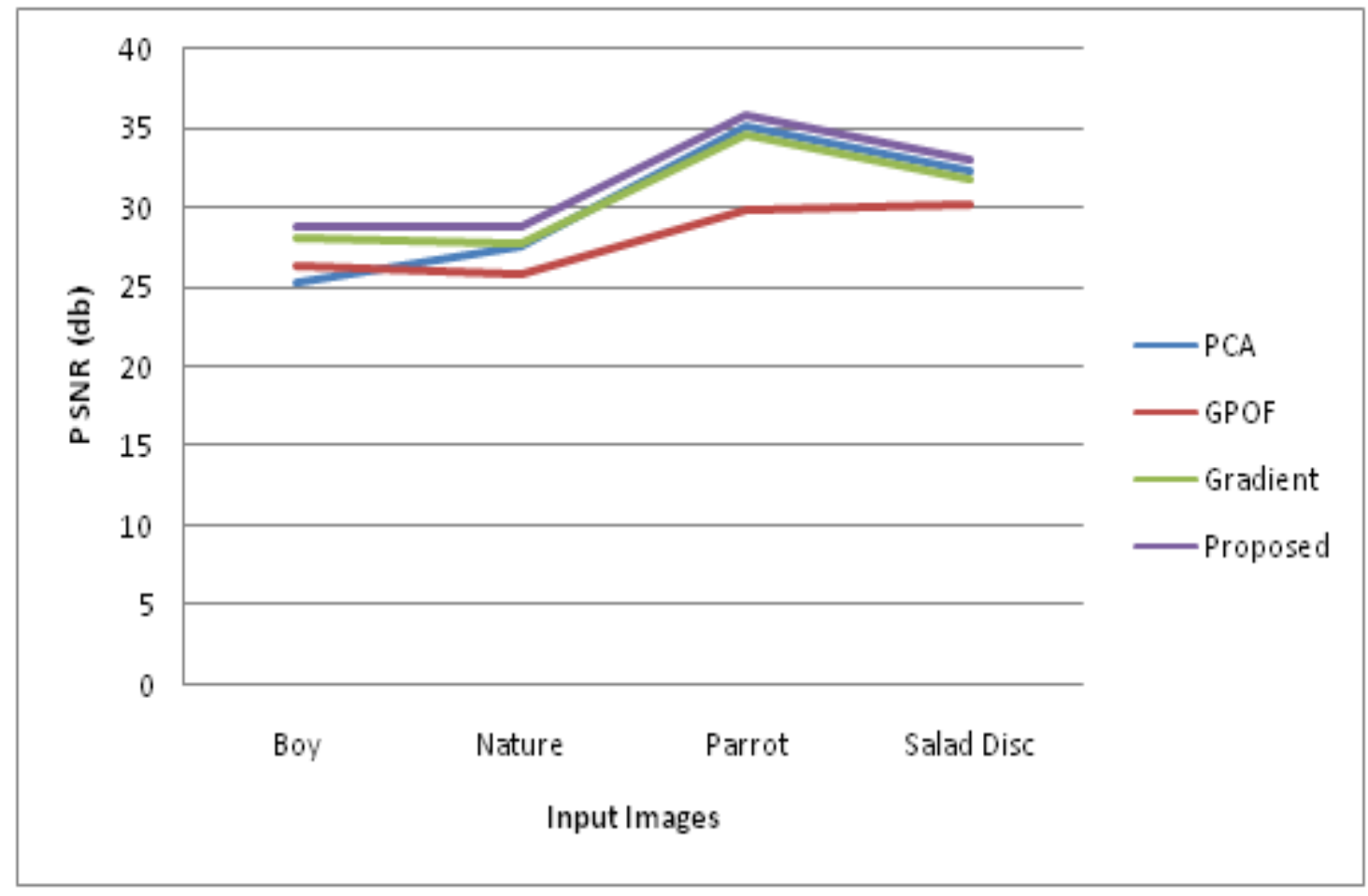

Figure 5: Input images vs. PSNR.

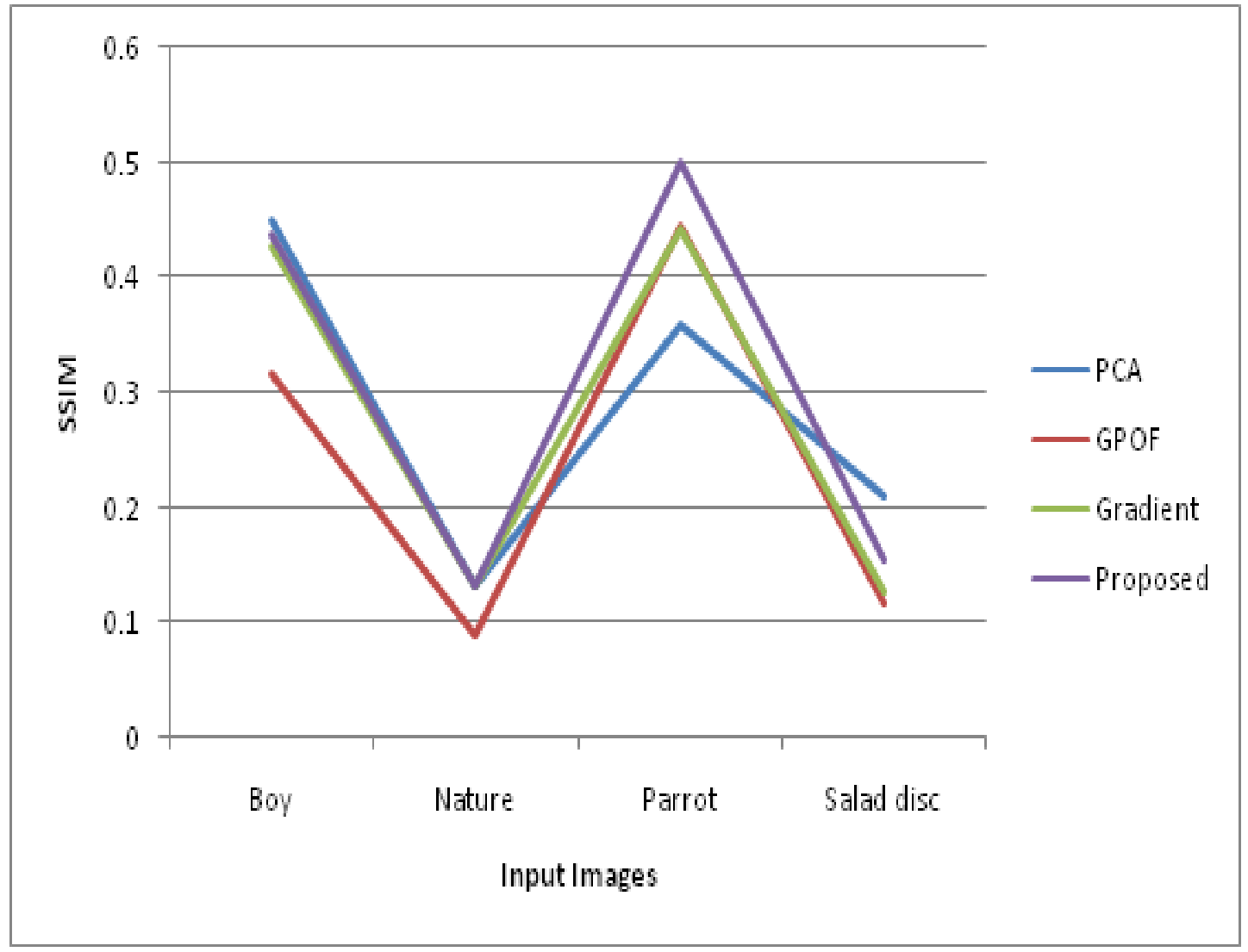

Figure 6: Input images vs. SSIM. 
Table 2. Image Quality measurement of PSNR, MSE, \& SSIM

\begin{tabular}{|c|c|c|c|c|c|}
\hline \multirow[t]{2}{*}{$\begin{array}{l}\text { Sr. } \\
\text { No. }\end{array}$} & \multirow[t]{2}{*}{ Image } & \multirow[t]{2}{*}{ Method } & \multicolumn{3}{|c|}{ Image Quality Measure } \\
\hline & & & MSE & PSNR & SSIM \\
\hline \multirow{4}{*}{1.} & \multirow{4}{*}{ Boy } & PCA & 25.1284 & 25.3579 & 0.4487 \\
\hline & & GPOF & 49.8052 & 26.3868 & 0.3156 \\
\hline & & Gradient & 33.1345 & 28.1568 & 0.4279 \\
\hline & & Proposed & 28.7818 & 28.7684 & 0.4360 \\
\hline \multirow{4}{*}{2.} & \multirow{4}{*}{ Nature } & PCA & 37.2978 & 27.6428 & 0.132 \\
\hline & & GPOF & 57.1187 & 25.7918 & 0.0891 \\
\hline & & Gradient & 36.5046 & 27.7361 & 0.1334 \\
\hline & & Proposed & 28.7339 & 28.7757 & 0.1329 \\
\hline \multirow{4}{*}{3.} & \multirow{4}{*}{ Parrot } & PCA & 19.9153 & 35.1389 & 0.3578 \\
\hline & & GPOF & 65.0586 & 29.9978 & 0.4449 \\
\hline & & Gradient & 22.1253 & 34.6819 & 0.4419 \\
\hline & & Proposed & 16.8433 & 35.8665 & 0.4999 \\
\hline \multirow{4}{*}{4.} & \multirow{4}{*}{$\begin{array}{c}\text { Salad } \\
\text { Disc }\end{array}$} & PCA & 37.7662 & 32.3598 & 0.2099 \\
\hline & & GPOF & 61.1471 & 30.2670 & 0.1177 \\
\hline & & Gradient & 42.7675 & 31.8197 & 0.1277 \\
\hline & & Proposed & 31.9765 & 33.0825 & 0.1567 \\
\hline
\end{tabular}

We compare three methods of image registration methods. Image quality measure using MSE and PSNR and plot the graph with the input images and performance parameter. In Figure 5: shows the graph with Input images vs. PSNR. The proposed results have been improved. In Figure 6: shows the graph of the input images vs. SSIM.

\section{CONCUSION}

This proposed techniques a combination of image registration methods GPOF and Gradient method for the image super resolution. Our proposed first GPOF registration is to measure the translation parameter and allows to large pixel motion but keeping the image neighborhood relatively small. And it can also reduce the complex process to high speed up the algorithm and apply the Gradient Method, which we can get the one output image, which achieves better result with the minor image movement. At last for image super resolution using image interpolation algorithms using Discrete Wavelet Transform, which gives the high resolution image. And we compares image registration methods with the proposed method, we gives the better results. We measure image quality using MSE, PSNR and SSIM with the original image and reconstructed image or high resolution image. MSE, PSNR and SSIM indicate that proposed algorithm give better results.

\section{REFERENCES}

[1] S. C. Park, M. K. Park, and M. G. Kang, "Superresolution image reconstruction: A technical review," IEEE Signal Processing Mag., vol. 20, pp. 21-36, May 2003.

[2] S. Chaudhuri, Ed., Super-Resolution Imaging. Norwell, MA: Kluwer, 2001.
[3] Lan Zhang; Hua Zhang; Simiao Zhang; YanbingXue, "Multi-frame image super-resolution reconstruction based on GPOF registration and L1-norm," Natural Computation (ICNC), 2010 Sixth International Conference on , vol.7, no., pp.3601,3604, 10-12 Aug. 2010

[4] Sasatani, S.; Xian-Hua Han; Yen-wei Chen, "Image registration using PCA and gradient method for superresolution imaging," Software Engineering and Data Mining (SEDM), 2010 2nd International Conference on , vol., no., pp.631,634, 23-25 June 2010

[5] Ping-Sing Tsai, Tinku Acharya," Image Up-Sampling Using Discrete Wavelet Transform", 9th JCIS 2006, 8 to 11 October 2006

[6] Simon Baker, Takeo Kanade, "Super-resolution optical flow", Technical Report CMU.

[7] Nan Zhao; Cuihua Li; Hua Shi; Chen Lin, "Multi-Frame Image Super-Resolution Based on Regularization Scheme," Control, Automation and Systems Engineering (CASE), 2011 International Conference on , vol., no., pp.1,4, 30-31 July 2011

[8] S. Farsiu, M. D. Robinson, M. Elad, and P. Milanfar, "Fast and robust Multi-frame super resolution", IEEE Trans. Image Process., vol. 13, no.10, pp. 1327-1344, 2004.

[9] Chidananda Murthy, M.V.; Yallapurmath, V.; Kurian, M. Z.; Guruprasad, H. S., "Design and implementation of interpolation algorithms for image super resolution," Communication Systems, Networks \& Digital Signal Processing (CSNDSP), 2012 8th International Symposium on, vol., no., pp.1,6, 18-20 July 2012

[10] Vishal R. Jaiswal, Suhas H. Patil, Girish P. Potdar, ShrishailT.Patil," Multi-frame Image Super-ResolutionA Comparison",(IJCSE) International Journal On Computer Science and Engineering, Vol. 02, No. 09, 2010, ISSN 0975-3397

[11] Xuelong Li, Yanting Hu, Xinbo Gao, et.al, "A multiframe image super-resolution method". IEEE Transactions on Signal Processing 90, pp.405-414, 2010.

[12] Turgay Celik, Kai-Kuang Ma, "Fast Object-based Image Registration Using Principal Component Analysis for Super-resolution Imaging", 5th International Conference on Visual Information Engineering, pp.705-710, 2008.

[13] Z. Wang, A. C. Bovik, H. R. Sheikh and E. P. Simoncelli, "Image quality assessment: From error visibility to structural similarity", IEEE Trans. Image Process., vol. 13, no. 4, pp. 600-612, 2004.

[14] Yusra A. Y. Al-Najjar, Dr. Der Chen Soong," Comparison of Image Quality Assessment: PSNR, HVS, SSIM, UIQI ", International Journal of Scientific \& Engineering Research, Volume 3, Issue 8, August-2012 1 ISSN 2229-5518

[15] R. Tsai, T. Huang,"Multi-frame image restoration and registration, in: Advances in Computer Vision and Image Processing, vol. 1, no. 2, JAI Press Inc., Greenwich, CT, 1984, pp. 317-339.

[16] Nelson, K.; Bhatti, A.; Nahavandi, S., "Performance Evaluation of Multi-Frame Super-Resolution Algorithms," Digital Image Computing Techniques and Applications (DICTA), 2012 International Conference on ,vol., no., pp.1,8, 3-5 Dec. 2012 